\title{
The clinical effects of combining postural exercises with chest physiotherapy in cystic fibrosis: A single-blind, randomized-controlled trial
}

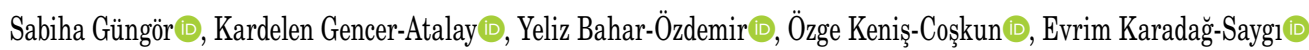 \\ Department of Physical Medicine and Rehabilitation, Marmara University School of Medicine, Istanbul, Turkey
}

Received: September 08, 2019 Accepted: February 25, 2020 Published online: March 04, 2021

\begin{abstract}
Objectives: This study aims to investigate the effects of postural exercises as an adjunct to chest physiotherapy program on respiratory function, exercise tolerance, quality of life (QoL), and postural stability in patients with cystic fibrosis (CF).

Patients and methods: In this single-blind, randomized-controlled trial, 19 pediatric CF patients (11 males, 8 females; mean age: 9.36 years; range, 6 to 14 years) were randomly allocated to chest physiotherapy and postural exercise program (Group 1, n=10) or chest physiotherapy program alone (Group 2, n=9) between March 2017 and October 2017. Respiratory functions were assessed with pulmonary function tests, whereas exercise tolerance with the Modified Shuttle Test (MST), quality of life with the Cystic Fibrosis Questionnaire-Revised Child Version (CFQR), and postural stability with the Limits of Stability Test (LOS). All tests were performed before treatment and six weeks, three months, and six months after treatment.

Results: Respiratory functions were improved in both groups; however, these changes were not statistically significant. The MST increased after treatment in both groups $(\mathrm{p}<0.001$ and $\mathrm{p}=0.003$ respectively), without a significant difference between the groups. Emotional function and treatment difficulties subdomains in CFQR were significantly increased only in the group with postural exercises ( $\mathrm{p}<0.05)$.

Conclusion: The postural exercise program in addition to chest physiotherapy in pediatric CF patients whose postural changes were not taken place did not cause significant changes in respiratory function, exercise tolerance, and postural stability; however, it affected the emotional state well and improved the compliance with the treatment.
\end{abstract}

Keywords: Chest physiotherapy, cystic fibrosis, postural exercises.

Cystic fibrosis (CF) is an autosomal recessive disease which mainly affects the respiratory system. ${ }^{[1]}$ The life expectancy for CF has dramatically increased in the recent decade with advancements in medical care. ${ }^{[2]}$ Pulmonary complications can be reduced and prevented by a regular chest physiotherapy program that mainly involves bronchial hygiene techniques. However, with the increased life expectancy, most of adults with CF have postural deformities, which cannot be prevented by conventional chest physiotherapy alone. Postural deformities in these population are claimed to be caused by muscle imbalance due to increased work of breathing as lung disease progresses, and associated with deteriorated pulmonary function, back pain, and impaired quality of life (QoL) ${ }^{[3]}$ In a study, it has been shown that none of the children with CF under the age of five years had a postural deformity; nevertheless, all of the patients above 13 had increased thoracic kyphosis, and some had accompanying scoliosis and chest abnormalities. ${ }^{[4]}$ Postural exercises

\footnotetext{
Corresponding author: Kardelen Gencer-Atalay, MD. Marmara Üniversitesi İstanbul Pendik Eğitim ve Araştırma Hastanesi, Fiziksel Tip ve Rehabilitasyon Anabilim Dall, 34899 Üst Kaynarca, Pendik, İstanbul, Türkiye.

e-mail: kardelengencer@gmail.com controlled trial. Turk J Phys Med Rehab 2021;67(1):91-98.
} 
which started before the deformities develop are thought to be preventive. Therefore, as rehabilitation professionals, we have begun to implement exercise therapies to rehabilitation programs for providing a complete approach to patients with CF in recent years.

The muscles that are involved in the stabilization of the body are also involved in respiration. ${ }^{[5]}$ Thus, it is assumed that postural exercises in children with CF would increase respiratory function, exercise tolerance, and QoL with preventing postural deformities. Although these assumptions are reasonable and understandable, there are no data about the effectiveness of postural exercise in children with CF.

In the present study, we hypothesized that the addition of a postural exercise program to a chest physiotherapy program would be effective in improving respiratory function, exercise tolerance, QoL, and postural stability compared to chest physiotherapy alone. We, therefore, aimed to evaluate the effects of additional postural exercises on these aspects in patients with CF.

\section{PATIENTS AND METHODS}

This single-blind, prospective, randomizedcontrolled study was conducted at Marmara University School of Medicine University, Physical Medicine and Rehabilitation Department between March 2017 and October 2017. Inclusion criteria were as follows: age between 6 to 14 years; having a diagnosis of $\mathrm{CF}$, and forced expiratory volume in $1 \mathrm{sec}$ (FEV1) greater than $30 \%$ of predicted. Exclusion criteria were as follows: presence of cor pulmonale, history of spinal fracture, currently being under intravenous (IV) medication, and severe gastroesophageal reflux. A total of 22 patients who met the inclusion criteria were included in the study. Two of them (one in each group) were lost to follow-up. Another patient was hospitalized due to an acute exacerbation with IV medication need and the treatment program was discontinued. Finally, a total of 19 pediatric CF patients (11 males, 8 females; mean age: 9.36 years; range, 6 to 14 years) were included (Figure 1). A written informed consent was obtained from each patient. The study protocol was approved by the Marmara University School of Medicine Ethics

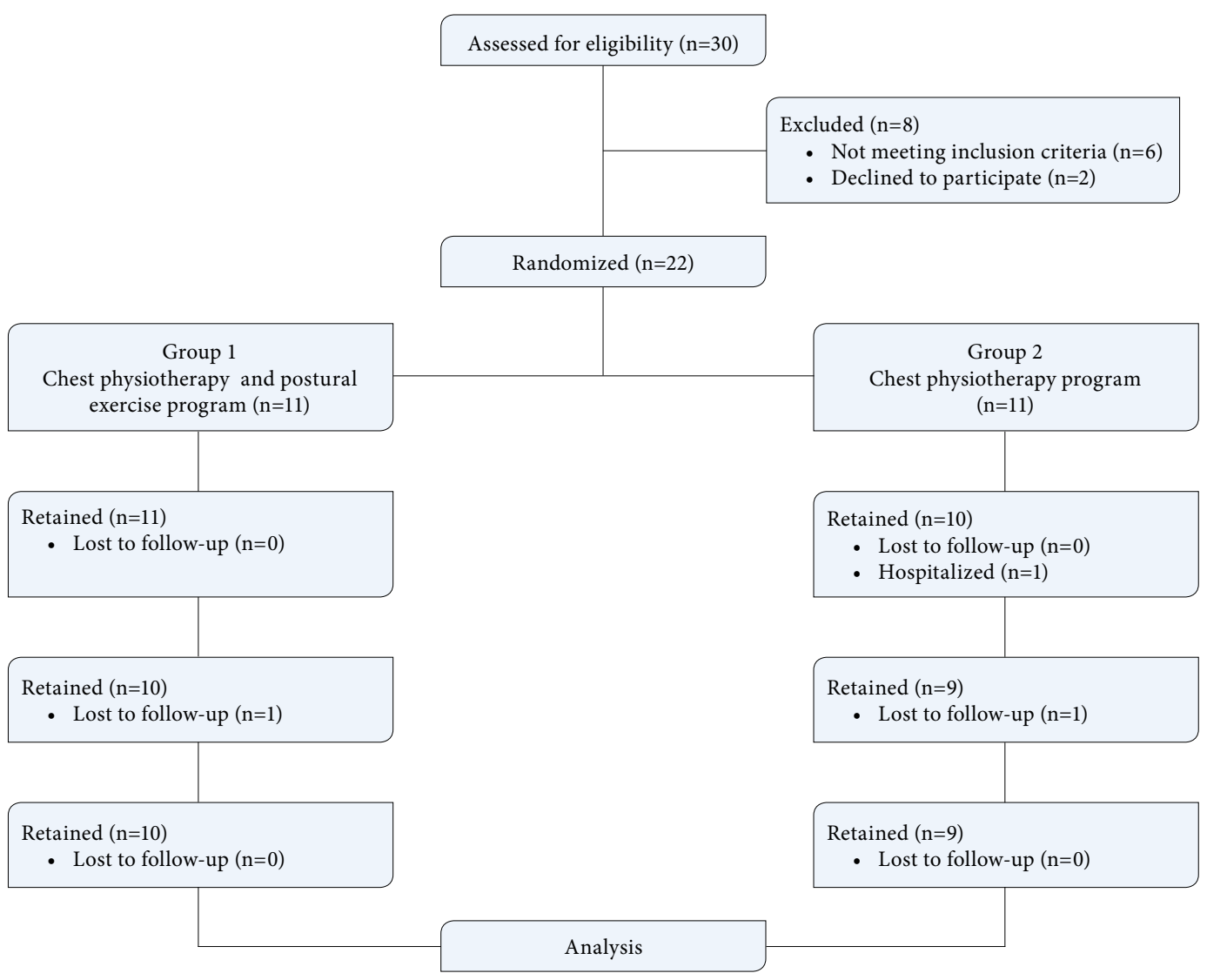

Figure 1. CONSORT diagram. 
Committee (Approval No: 09.2015.287). The study was conducted in accordance with the principles of the Declaration of Helsinki. This study was registered with ClinicalTrials.gov (NCT03295201).

The patients who did not previously participate in a structured chest physiotherapy program and other techniques regularly before the study were equally randomized into two groups according to the sealed opaque envelope system with blocking. One group was planned to be treated six weeks with chest physiotherapy and postural exercise program (Group 1, $\mathrm{n}=10$ ), while the other group was treated with chest physiotherapy program alone (Group 2, n=9). Both groups received treatment by a single physiotherapist. If a patient could not attend to the treatment program due to interruptions such as an acute exacerbation, he/she was removed from the final analysis. Respiratory function was assessed with FEV1, forced vital capacity (FVC), FEV1/FVC, and peak expiratory flow (PEF), whereas exercise tolerance with the Modified Shuttle Test (MST), QoL with the Cystic Fibrosis QuestionnaireRevised Child Version (CFQR) and postural stability with The NeuroCom Balance Master ${ }^{\oplus}$ device (NeuroCom International, Clackamas, OR, USA) Limits of Stability Test (LOS). All tests were performed before treatment and six weeks, three months, and six months after treatment. The Cobb and modified Cobb angles were measured on radiographic examinations to assess scoliosis and thoracic kyphosis before treatment and six months after treatment. All evaluations were performed by a blinded independent rehabilitation specialist. Data were collected at Marmara University School of Medicine, Physical Medicine and Rehabilitation Department, Pediatric Rehabilitation Clinic.

\section{Treatment}

\section{Chest physiotherapy program}

Active cycle of breathing techniques (ACBTs) was performed for chest physiotherapy. This is a commonly used method for secretion removal in $\mathrm{CF}$ patients. Every ACBT cycle consists of a sequence of breathing control, thoracic expansion exercises and forced expiration and cycles are repeated, until the secretion is completely discharged. ${ }^{[6]}$ The ACBT was applied by a therapist once per week for six weeks. This interval between chest physiotherapy programs was arranged to provide isolation of each patient according to infection control recommendations. ${ }^{[7]}$ Therefore, a daily schedule was given for the other days of the week once per day to ensure the permanence of the treatment. After the treatment period, families were encouraged with weekly telephone calls to continue their child's chest physiotherapy program for six months.

\section{Postural exercise program}

Thoracic vertebra mobilization, pectoral stretching, scapula and thoracic extensor muscle strengthening, and core stability exercises were applied by a therapist once per a week for six weeks. A daily schedule was also given including pectoral stretching and core stabilization exercises for the other days of the week once per day to ensure the permanence of treatment. After the treatment period, families were encouraged with weekly telephone calls to continue their child's exercise program for six months.

\section{Assessment tools}

\section{Modified Shuttle Test}

The MST is a simple test which measures exercise tolerance and cardiorespiratory status in children with CF. The test has 15 levels, and the patient is asked to walk rapidly between two fixed objects within a 10 -meter distance, starting with normal walking speed and increasing the pace at the beginning of each min (level). An audio signal is used to maintain the required speed. The test is continued until the end of 15 levels or is ended when the patient cannot keep pace with audio signal any longer or feels tired. Peripheral oxygen saturation $\left(\mathrm{SpO}_{2}\right)$ is measured during the test period using a pulse oximeter, and the interruption criteria were determined as $\mathrm{SpO}_{2}$ lower than $75 \%{ }^{[8,9]}$ Maximum walking distance expressed in meters was used for analysis as an outcome.

\section{Cystic Fibrosis Questionnaire-Revised}

The CFQR is the most commonly used QoL measurement tool in patients with $\mathrm{CF}$ and is found to be valid and reliable in Turkish. The child version of this test consists of 35 questions about physical function, emotional function, social function, body appearance, eating disorders, treatment difficulties, respiratory and digestive symptoms. The total score is calculated between 0 and 100, and higher scores indicate the better condition. ${ }^{[10]}$

\section{Limits of Stability Test}

This is a test of NeuroCom Balance Master ${ }^{\circledR}$ device that consists of a $18 \times 60$-inch pressure platform and a computer system connected to the platform. The patient is asked to stand on the platform barefoot and watch the image which can be moved by trunk movement 
on the computer the monitor, and with a command, move the image toward to target point which is in eight different directions. Reaction time, movement velocity, endpoint excursion, maximum excursion, and direction control parameters are calculated for each direction during these trunk movements. ${ }^{[1]}$

\section{Statistical analysis}

To depict a $20 \%$ increase of the distance covered in MST by patients, the projected sample size was calculated as approximately eight patients and eight controls with an alpha $=0.05$ and power $=0.80 .{ }^{[8]}$ Statistical analysis was calculated using the IBM SPSS for Windows version 20.0 software (IBM Corp., Armonk, NY, USA). Descriptive data were expressed in mean \pm standard deviation (SD), median (min-max) or number and frequency. Normal distribution of quantitative values was assessed by histogram, Q-Q graph, and Shapiro-Wilk test. Since the distribution was not normal, the Mann-Whitney $\mathrm{U}$ test was used to compare the measurement values between the two groups, and the Friedman test was used to compare the intra-group parameters, while the Wilcoxon test was used to compare pre-treatment and post-treatment sixth-week, third-month, and sixth-month values separately. The Bonferroni correction was considered statistically significant at $\mathrm{p}<0.010$ for evaluating the results of the Wilcoxon test. Otherwise, a $p$ value of $<0.05$ was considered statistically significant.

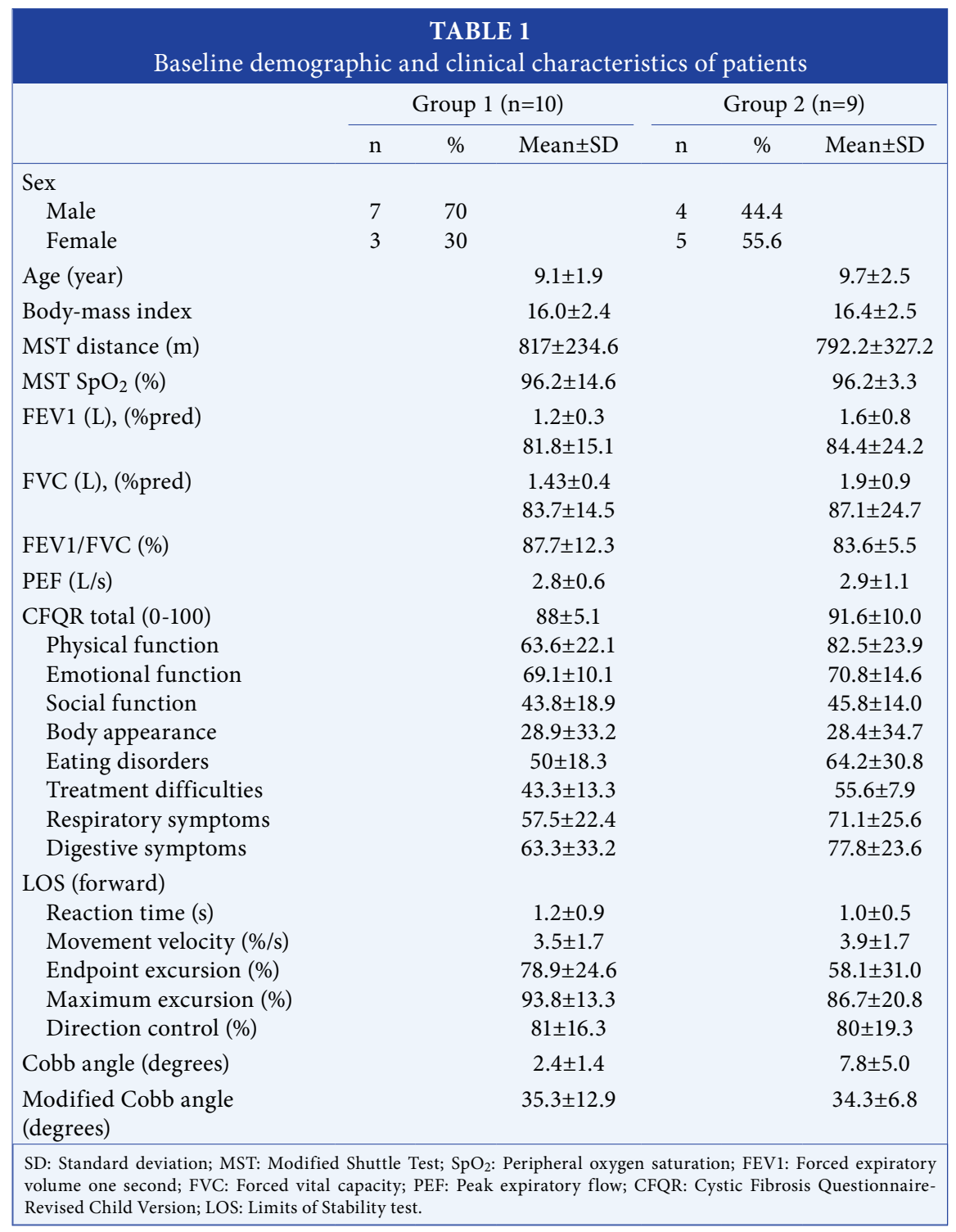




\section{RESULTS}

Baseline demographic and clinical characteristics of the patients are shown in Table 1. There was no statistically significant difference in the baseline demographic characteristics between the patient groups. None of the patients in both groups had scoliosis or increased thoracic kyphosis. Except for the patients excluded from the analysis, none of the patients experienced an acute exacerbation leading to treatment discontinuation.
None of the patients complained about pain before or during the treatment. The MST distance significantly increased at six weeks in both groups, and it continued to increase at three and six months (Table 2). Respiratory function tests were also improved after treatment in both groups, although these changes were not statistically significant (Table 3). The CFQR emotional function and treatment difficulties subdomains significantly increased at the end of six weeks in Group 1.

\section{TABLE 2}

Intra-group and inter-group comparisons for exercise tolerance

\begin{tabular}{|c|c|c|c|c|c|c|c|}
\hline \multirow[b]{2}{*}{ MST m } & \multicolumn{3}{|c|}{ Group $1(\mathrm{n}=10)$} & \multicolumn{3}{|c|}{ Group $2(n=9)$} & \multirow[b]{2}{*}{$p \ddagger$} \\
\hline & Median & IR & $p \dagger$ & Median & IR & $p^{\dagger}$ & \\
\hline Before treatment ${ }^{1}$ & 860 & 412.5 & $\mathrm{p}^{1-2}=0.007^{\star}$ & 730 & 545 & $\mathrm{p}^{1-2}=0.008^{*}$ & 0.838 \\
\hline Sixth weeks after treatment ${ }^{2}$ & 990 & 377.5 & $\mathrm{p}^{1-3}=0.005^{\star}$ & 760 & 830 & $\mathrm{p}^{1-3}=0.008^{\star}$ & 0.806 \\
\hline Third months after treatment ${ }^{3}$ & 1075 & 402 & $\mathrm{p}^{1-4}=0.005^{\star}$ & 960 & 790 & $\mathrm{p}^{1-4}=0.008^{\star}$ & 0.653 \\
\hline Sixth months after treatment ${ }^{4}$ & 1235 & 365 & $\begin{array}{l}\mathrm{p}^{2-3}=0.005^{\star} \\
\mathrm{p}^{3-4}=0.02\end{array}$ & 960 & 705 & $\begin{array}{l}\mathrm{p}^{2-3}=0.767 \\
\mathrm{p}^{3-4}=0.4\end{array}$ & 0.390 \\
\hline$P$ value (Friedman test) & \multicolumn{2}{|c|}{$<0.001$} & & \multicolumn{2}{|c|}{$0.003^{* *}$} & & \\
\hline
\end{tabular}

\section{TABLE 3}

Intra-group and inter-group comparisons for respiratory functions

\begin{tabular}{|c|c|c|c|c|c|}
\hline & \multicolumn{2}{|c|}{ Group $1(\mathrm{n}=10)$} & \multicolumn{2}{|c|}{ Group $2(\mathrm{n}=9)$} & \multirow[b]{2}{*}{$p$} \\
\hline & Median & IR & Median & IR & \\
\hline \multicolumn{6}{|l|}{ FEV1 L (\%pred) } \\
\hline Before treatment & $1.22(86)$ & $0.47(26)$ & $1.17(88.5)$ & $1.18(33.5)$ & 0.630 \\
\hline Sixth weeks after treatment & $1.31(90.5)$ & $0.69(37.75)$ & $1.35(86)$ & $1.22(19)$ & 0.368 \\
\hline Third months after treatment & $1.42(91.5)$ & $0.79(22)$ & $1.04(85)$ & $1.27(19)$ & 0.643 \\
\hline Sixth months after treatment & $1.56(88.5)$ & $0.77(8.75)$ & $1.6(95.5)$ & $1.75(49.25)$ & 0.873 \\
\hline$P$ value (Friedman test) & \multicolumn{2}{|c|}{0.272} & \multicolumn{2}{|c|}{0.532} & \\
\hline \multicolumn{6}{|l|}{ FVC L (\%pred) } \\
\hline Before treatment & $1.29(88)$ & $0.66(10)$ & $1.60(87)$ & $1.46(33.5)$ & 0.310 \\
\hline Sixth weeks after treatment & $1.42(94)$ & $0.72(19)$ & $1.01(75.5)$ & $1.01(54)$ & 0.157 \\
\hline Third months after treatment & $1.61(94)$ & $0.83(21.25)$ & $2.27(99)$ & $1.78(36)$ & 0.685 \\
\hline Sixth months after treatment & $1.82(93.5)$ & $0.84(14)$ & $1.82(95)$ & $1.95(41.5)$ & 0.749 \\
\hline$P$ value (Friedman test) & \multicolumn{2}{|c|}{0.054} & \multicolumn{2}{|c|}{0.392} & \\
\hline \multicolumn{6}{|l|}{ FEV1/FVC (\%) } \\
\hline Before treatment & 87.4 & 23.4 & 86.8 & 10.45 & 0.847 \\
\hline Sixth weeks after treatment & 87.8 & 13.1 & 85.1 & 18.38 & 0.906 \\
\hline Third months after treatment & 85.95 & 15.38 & 83.3 & 12 & 0.563 \\
\hline Sixth months after treatment & 83.3 & 7.1 & 85.95 & 7.2 & 0.150 \\
\hline$P$ value (Friedman test) & \multicolumn{2}{|c|}{0.440} & \multicolumn{2}{|c|}{0.615} & \\
\hline \multicolumn{6}{|l|}{$\mathrm{PEF} \mathrm{L/s}$} \\
\hline Before treatment & 2.52 & 0.81 & 2.91 & 0.99 & 0.958 \\
\hline Sixth weeks after treatment & 3.12 & 1.55 & 2.08 & 1.7 & 0.125 \\
\hline Third months after treatment & 2.72 & 1.44 & 2.62 & 2.93 & 0.643 \\
\hline Sixth months after treatment & 3.35 & 1.76 & 2.77 & 2.37 & 0.522 \\
\hline$P$ value (Friedman test) & 0.552 & & 0.135 & & \\
\hline
\end{tabular}




\begin{tabular}{|lcccccccc}
\multicolumn{7}{c}{ TABLE } \\
Intra-group and inter-group comparisons for CFQR emotional function and treatment difficulties subdomains
\end{tabular}

However, this change disappeared after the sixth week (Table 4). Other subdomains in Group 1 and all subdomains in Group 2 remained unchanged after treatment $(p>0.05)$. Also, the LOS test items in all directions remained unchanged after treatment in both groups ( $p>0.05)$. The Cobb and modified Cobb angles did not change at six months in either group ( $p>0.05)$. No adverse events were observed in either group during treatment or follow-up.

\section{DISCUSSION}

In the present study, postural exercises added to a conventional chest physiotherapy program increased treatment compliance and improved emotional status, while exercise tolerance, respiratory functions, and postural stability remained unchanged.

In patients with $\mathrm{CF}$, conventional chest physiotherapy improves respiratory functions, as demonstrated time and again in the literature. ${ }^{[12,13]}$ Similarly, in this study, although no statistical significance was reached, the conventional chest physiotherapy program increased respiratory functions in both groups. However, postural exercises did not add a significant benefit. This contradicts the common hypothesis of the bilateral relationship between respiratory and postural muscles which make postural muscles accessories in respiration. ${ }^{[14]}$ A previous study showed that thoracic kyphosis angle was correlated with FEV1 and vital capacity. ${ }^{[15]}$ However, Sansund et al. ${ }^{[16]}$ documented that, in patients with a high thoracic index, postural exercises added to a conventional chest physiotherapy program could decrease the thoracic index, but did not significantly change respiratory functions. Likewise, Schindel et al. ${ }^{[13]}$ showed that home-based aerobic exercise and stretching improved postural alignment without significant change in respiratory functions. Postural deformities increase with age in patients with $\mathrm{CF}^{\left[{ }^{[17]}\right.}$ The mean age of the study that conducted by Schindel et al. ${ }^{[13]}$ was four years older than the present study. One of the reasons that this study could not show any beneficial effects of postural exercises on respiratory functions is that the patients included in the study did not have spinal deformities. It must be kept in mind that, in an older patient population, the outcomes may differ.

In the current study, the MST distance increased after treatment in both groups, and this increase continued in the long-term. Although MST distance in children and adolescent has been shown to increase with age, ${ }^{[9]}$ the increase at six weeks and three months can be easily attributed to the effect of chest physiotherapy. However, the long-term increase in MST may be due to the natural effect of growing up, leading to the assumption of some contribution to the longer-term positive results. Doeleman et al. ${ }^{[18]}$ demonstrated a strong relationship between FEV1 
and MST distances in adult patients with CF. In the study by Cox et al,. ${ }^{[19]} 28$ patients with $\mathrm{CF}$ were admitted for pulmonary exacerbations and, after the administration of IV antibiotics, the MST distance improved significantly. There are existing data regarding the improvement of exercise tolerance after medical treatment. However, there are no available data regarding the effectiveness of chest physiotherapy programs on exercise tolerance. While FEV1 is an important follow-up criterion in the clinical setting of $\mathrm{CF}$, exercise tolerance tests may give better ideas about the actual functionality of the patient, which makes them more important in the rehabilitation setting. To the best of our knowledge, this study is the first to use exercise tolerance tests for the evaluation of functionality of CF patients.

In this study, none of the patients in both groups had apparent postural impairment as evidenced by imaging studies. The absence of change in postural alignment at six months is not sufficient to demonstrate the effectiveness or ineffectiveness of postural exercises on posture. However, this is beyond the scope of this study and a much more extended follow-up period would provide such a kind of effect. On the other hand, we aimed to examine the effect of postural exercises on postural stability. The LOS is a test which evaluates the distance and direction control of a person, when it is attempted to reach various directions by trunk movement. ${ }^{[1]}$ These parameters of LOS were shown to be improved with postural training, even in healthy young adults. ${ }^{[20]}$ Therefore, an improvement was expected with postural exercises in patients with CF, although they did not have apparent problems with their posture or postural stability. The lack of change in postural stability parameters in this study may be due to the intensity and frequency of the exercise program. However, since it is not advised for CF patients to frequently admit to the hospital due to colonization and infection risk, more than once a week could have increased the incidence of acute exacerbations. Future research can focus on homebased interventions and their effectiveness in this regard.

There were significant improvements after postural exercises in the emotional status and treatment difficulty subdomains of the CFQR. However, these changes did not last after six weeks, when the treatment program was ended. On the other hand, conventional chest physiotherapy alone did not change any parameters of QoL at any time. Similar to these findings, Schmidth et al. ${ }^{[21]}$ showed that, in adult patients with CF, an exercise program improved emotional status and treatment compliance subdomains. Mandal et al. ${ }^{[22]}$ investigated the effect of additional exercise program to conventional chest physiotherapy in 30 patients with bronchiectasis and showed that pulmonary physiotherapy alone could not change the QoL and exercise tolerance. In the study by Sansund et al., ${ }^{[16]}$ addition of postural exercises to a conventional chest, physiotherapy did not change the QoL in patients with CF. This may have resulted due to the fact that only physical function subdomain was investigated in the QoL measures in this study. These findings are consistent with our findings, indicating that postural exercises in the course of the therapy period improve emotional status and treatment compliance in patients with CF.

To the best of our knowledge, this is the first randomized-controlled study to investigate the effect of postural exercises added to a conventional chest physiotherapy program on respiratory functions, exercise tolerance, QoL, and postural stability in patients with CF. However, due to the nature of studies using exercise as therapy, it was designed as a single-blind study. This is an important bias factor. On the other hand, randomization process of patient selection, outcome assessor blinding, and no dropouts decrease the overall bias of this study. One of the main strengths of this study is its long-term follow-up duration, while limitations of this study can be stated as the relatively small sample size. Future studies with an increased patient population and a longer follow-up period would definitely show more concrete results about the effectiveness of such rehabilitation programs. In the setting of physiatry, an exercise regimen initiated early in the course of the disease has been shown to be effective in a spectrum of pathologies. ${ }^{[23-25]}$ Although CF should be no different, the data that would support the hypothesis of the importance of early intervention in this population are still lacking. A pivotal study can include patient groups who can and cannot attend to these programs and, therefore, the change in postural deformities in the long-term can be documented. Moreover, the improvements in the emotional state and treatment compliance can also have an additional positive effect on CF-related outcomes, such as pulmonary function. The effect of increased treatment compliance and improved emotional state should be investigated on its effect on the frequency of acute exacerbations and hospitalization need. Therefore, particularly through the adolescent to adulthood 
transition period, when the patient care also transits between different practitioners, long-term protocols with an extended duration of supervised postural exercise as the cornerstone of care would give us valuable information about the effectiveness of such programs. This is also essential for patient care and daily practice, as it must be remembered that CF is a chronic, lifelong condition in nature.

In conclusion, our study results indicate that postural exercises do not significantly improve respiratory functions, exercise tolerance, and postural stability in these young patients without apparent postural changes. However, postural exercise program significantly improves emotional status and treatment compliance, and chest physiotherapy improves exercise tolerance in these patients.

\section{Declaration of conflicting interests}

The authors declared no conflicts of interest with respect to the authorship and/or publication of this article.

\section{Funding}

The authors received no financial support for the research and/or authorship of this article.

\section{REFERENCES}

1. Edwards J, Clarke A, Greenop D. Adults with cystic fibrosis - responding to a new ageing population. Chronic Illn 2013;9:312-9.

2. Buzzetti R, Salvatore D, Baldo E, Forneris MP, Lucidi V, Manunza D, et al. An overview of international literature from cystic fibrosis registries: 1 . Mortality and survival studies in cystic fibrosis. J Cyst Fibros 2009;8:229-37.

3. Tattersall R, Walshaw MJ. Posture and cystic fibrosis. J R Soc Med 2003;96 Suppl 43:18-22.

4. Rawo T, Tramś M, Michalski P, Sands D. Postural defects in children with cystic fibrosis - preliminary report. Dev Period Med 2015;19:114-9.

5. Okuro RT, Côrrea EP, Conti PB, Ribeiro JD, Ribeiro MA, Schivinski CI. Influence of thoracic spine postural disorders on cardiorespiratory parameters in children and adolescents with cystic fibrosis. J Pediatr (Rio J) 2012;88:310-6.

6. Daniels T. Physiotherapeutic management strategies for the treatment of cystic fibrosis in adults. J Multidiscip Healthc 2010;3:201-12.

7. Saiman L, Siegel J. Infection control in cystic fibrosis. Clin Microbiol Rev 2004;17:57-71.

8. Coelho CC, Aquino Eda S, de Almeida DC, Oliveira GC, Pinto Rde C, Rezende IM, et al. Comparative analysis and reproducibility of the modified shuttle walk test in normal children and in children with cystic fibrosis. J Bras Pneumol 2007;33:168-74.

9. Lanza Fde C, Zagatto Edo P, Silva JC, Selman JP, Imperatori $\mathrm{TB}$, Zanatta DJ, et al. Reference equation for the incremental shuttle walk test in children and adolescents. J Pediatr 2015;167:1057-61.
10. Yuksel H, Yilmaz O, Dogru D, Karadag B, Unal F, Quittner AL. Reliability and validity of the Cystic Fibrosis QuestionnaireRevised for children and parents in Turkey: cross-sectional study. Qual Life Res 2013;22:409-14.

11. Ku PX, Abu Osman NA, Wan Abas WAB. The limits of stability and muscle activity in middle-aged adults during static and dynamic stance. J Biomech 2016;49:3943-8.

12. Warnock L, Gates A. Chest physiotherapy compared to no chest physiotherapy for cystic fibrosis. Cochrane Database Syst Rev 2015;2015:CD001401.

13. Schindel CS, Hommerding PX, Melo DA, Baptista RR, Marostica PJ, Donadio MV. Physical exercise recommendations improve postural changes found in children and adolescents with cystic fibrosis: a randomized controlled trial. J Pediatr 2015;166:710-6.e2.

14. Ashbrook JE, Shacklady C, Johnson S, Yeowell G, Goodwin PC. Is there an association between back pain and stress incontinence in adults with cystic fibrosis? A retrospective cross-sectional study. J Cyst Fibros 2018;17:78-82.

15. Mellin G, Harjula R. Lung function in relation to thoracic spinal mobility and kyphosis. Scand J Rehabil Med 1987;19:89-92.

16. Sandsund CA, Roughton M, Hodson ME, Pryor JA. Musculoskeletal techniques for clinically stable adults with cystic fibrosis: a preliminary randomised controlled trial. Physiotherapy 2011;97:209-17.

17. Tattersall R, Walshaw MJ. Posture and cystic fibrosis. J R Soc Med 2003;96 Suppl 43:18-22.

18. Doeleman WR, Takken T, Bronsveld I, Hulzebos EH. Relationship between lung function and Modified Shuttle Test performance in adult patients with cystic fibrosis: a cross-sectional, retrospective study. Physiotherapy 2016;102:184-8.

19. Cox NS, Follett J, McKay KO. Modified shuttle test performance in hospitalized children and adolescents with cystic fibrosis. J Cyst Fibros 2006;5:165-70.

20. Siriphorn A, Chamonchant D, Boonyong S. Exercise using mung bean bag improves balance in healthy young adults. Physiother Theory Pract 2016;32:603-11.

21. Schmidt AM, Jacobsen U, Bregnballe V, Olesen HV, Ingemann-Hansen $\mathrm{T}$, Thastum $\mathrm{M}$, et al. Exercise and quality of life in patients with cystic fibrosis: A 12-week intervention study. Physiother Theory Pract 2011;27:548-56.

22. Mandal P, Sidhu MK, Kope L, Pollock W, Stevenson LM, Pentland JL, et al. A pilot study of pulmonary rehabilitation and chest physiotherapy versus chest physiotherapy alone in bronchiectasis. Respir Med 2012;106:1647-54.

23. Novak I, Cusick A. Home programmes in paediatric occupational therapy for children with cerebral palsy: Where to start? Australian Occupational Therapy Journal 2006;53:251-64.

24. Rasmussen L, Justice D, Chang KW, Nelson VS, Yang LJ. Home exercise DVD promotes exercise accuracy by caregivers of children and adolescents with brachial plexus palsy. PM R 2013;5:924-30.

25. Crytzer TM, Dicianno BE, Kapoor R. Physical activity, exercise, and health-related measures of fitness in adults with spina bifida: a review of the literature. PM R 2013;5:1051-62. 